\title{
Testosterone level and mortality in elderly men with systolic chronic heart failure
}

\begin{abstract}
Hai-Yun $\mathrm{Wu}^{1}$, Xiao-Fei Wang ${ }^{2}$, Jun-Hua Wang ${ }^{3}$ and Jiang-Yuan $\mathrm{Li}^{4}$
Previous studies on the prognostic significance of serum levels of androgens in patients with chronic heart failure (CHF) have yielded conflicting results. The aim of this study was to examine the relationship between serum concentration of testosterone and mortality in men with systolic CHF. A total of 175 elderly men (age $\geqslant 60$ years) with CHF were recruited. Total testosterone (TT) and sex hormone-binding globulin (SHBG) were measured, and estimated free testosterone (eFT) was calculated. The median follow-up time was 3.46 years. Of these patients, 17 had a TT level below $8 \mathrm{nmol} \mathrm{I}^{-1}\left(230 \mathrm{ng} \mathrm{dl}^{-1}\right), 27 \mathrm{had}$ an eFT level below $0.225 \mathrm{nmol} \mathrm{I}^{-1}\left(65 \mathrm{pg} \mathrm{ml}^{-1}\right)$ and 12 had both. Using the age-specific tenth percentiles of TT and eFT in healthy men in our laboratory as cutoff points, the prevalences of TT and eFT deficiency was $21.7 \%(38 / 175)$ and $27.4 \%(48 / 175)$, respectively. Both TT and eFT were inversely associated with left ventricular ejection fraction (LVEF) and N-terminal pro-brain natriuretic peptide (NT-pro-BNP) (all $\boldsymbol{P}<0.01$ ). Kaplan-Meier curves for patients in low, medium and high tertiles according to TT and eFT level showed significantly different cumulative survival rate (both $\boldsymbol{P}<0.01$ by log-rank test). However, after adjustment for clinical variables, there were no significant associations of either TT or eFT levels with survival time $(\mathrm{OR}=0.97,95 \% \mathrm{Cl}: 0.84-1.12$, $P=0.28$ and $\mathrm{OR}=0.92,95 \% \mathrm{Cl}: 0.82-1.06, P=0.14$, respectively). Our study showed that levels of TT and eFT are commonly decreased in elderly patients with systolic CHF and related to disease severity, but they are not independent predictors for mortality.
\end{abstract}

Asian Journal of Andrology (2011) 13, 759-763; doi:10.1038/aja.2011.26; published online 20 June 2011

Keywords: free testosterone; heart failure; prognosis; total testosterone

\section{INTRODUCTION}

Chronic heart failure (CHF) is a major health problem throughout the world. ${ }^{1}$ In China, the prevalence of CHF was estimated to be $0.9 \%$ for the general population, $0.7 \%$ and $1.0 \%$ for the men and the women, respectively, and the prevalence of CHF increased substantially with age. ${ }^{2}$ Previous studies have shown that androgen deficiency in men with CHF, and levels of testosterone, dehydroepiandrosterone and its sulfate have been reported to be decreased with increasing severity of heart failure. ${ }^{3-6}$ It has been suggested that a low testosterone level may represent one of the factors contributing to the anabolic/catabolic imbalance characteristically present in many patients with advanced $\mathrm{CHF}^{4,6-9}$ and recent interventional studies have indicated that physiological testosterone supplementation increases functional capacity and muscle performance in both male ${ }^{10-13}$ and female ${ }^{14}$ patients with CHF. However, until now, only a few studies on the prognostic significance of serum levels of androgens in CHF patients have been published and yielded conflicting results. ${ }^{15,16}$ The purpose of the present study was to evaluate prospectively the prevalence and prognostic consequences of androgens deficiencies in a cohort of elderly male patients with systolic CHF.

\section{MATERIALS AND METHODS}

Study population

From 1 March 2005 to 31 May 2007, consecutive elderly male patients with CHF and reduced left ventricular ejection fraction (LVEF) who attended the outpatient clinics in three cardiology departments (Chinese PLA Genearal Hospital, the 306th Hospital of Chinese PLA, and the Chinese PLA Air Force Hospital, Beijing, China) were enrolled in this prospective study. The criteria for study inclusion were: (i) age 60 years or over; (ii) a documented history of CHF for at least 6 months; (iii) LVEF of $45 \%$ or less as assessed by echocardiography; and (iv) clinical stability and unchanged medications for at least 1 month preceding the study. Patients with cirrhosis and alcoholism were excluded because these conditions can cause low testosterone concentrations. ${ }^{17}$

During the recruitment period, 181 patients who were suitable for the study and who agreed to participate were identified. In six patients, blood analyses on hormones were not feasible due to storage failure; thus, the current report refers to 175 subjects.

The study protocol was approved by the ethics committees of the participating hospitals and all patients gave written informed consent.

${ }^{1}$ Institute of Geriatric Cardiology, Chinese PLA General Hospital, Beijing 100853, China; ${ }^{2}$ Department of Cardiology, the 306th Hospital of Chinese PLA, Beijing 100101, China; ${ }^{3}$ Department of Cardiology, Chinese PLA Airforce General Hospital, Beijing 100036, China and ${ }^{4}$ Department of Endocrinology, Chinese PLA General Hospital, Beijing 100853, China 
Serum concentrations of androgens and other chemical measurements

Venous blood samples were taken in the morning between 07:00 and 08:00 following an overnight fast and after a supine rest of at least $15 \mathrm{~min}$. After centrifugation, serum blood was collected and frozen at $-70{ }^{\circ} \mathrm{C}$ until it was analyzed. Serum concentrations of total testosterone (TT) was measured by electrochemiluminescence (ACS:180; Bayer, Leverkusen, Germany). The inter-assay and intraassay variability coefficients for TT were $6.5 \%$ and $7.0 \%$, respectively. Serum level of sex hormone-binding globulin (SHBG) was measured with ELISA (BL, Hamburg, Germany), and the interassay and intra-assay variability coefficients were $5.2 \%$ and $3.0 \%$, respectively, as described previously. ${ }^{18}$ Serum level of estimated free testosterone (eFT) was calculated with the validated equation of Vermeulen et al. ${ }^{19}$ Serum N-terminal pro-brain natriuretic peptide (NT-pro-BNP) was measured using an enzyme-linked immunosorbent assay microtiter plate (Roche Diagnostics, Basel, Switzerland). All measurements were performed at the Laboratory of the Endocrinology, Department of Chinese PLA General Hospital and by staff unaware of the clinical data. In our laboratory, the tenth percentiles of TT were $13.88 \mathrm{nmol} \mathrm{l}^{-1}\left(400.00 \mathrm{ng} \mathrm{dl}^{-1}\right)$ for healthy men aged 60-69 years and $12.35 \mathrm{nmol} \mathrm{l}^{-1}\left(356.00 \mathrm{ng} \mathrm{dl}^{-1}\right)$ for men aged 70 years or over, and the tenth percentiles of eFT were $0.22 \mathrm{nmol} \mathrm{l}^{-1}\left(6.20 \mathrm{ng} \mathrm{dl}^{-1}\right)$ for men aged 60-69 years and $0.19 \mathrm{nmol} \mathrm{l}^{-1}\left(5.80 \mathrm{ng} \mathrm{dl}^{-1}\right)$ for men aged 70 years or over. ${ }^{18}$ Estimated glomerular filtration rate was calculated using the formula from the Modification of Diet in Renal Disease Study Group. ${ }^{20}$ As comparison, we also used the criteria for initiation of testosterone treatment as proposed by the current recommendations $^{21,22}$ for data analysis.

\section{Mortality data}

Patient status (dead or alive) was ascertained between May and July 2010 by contacting the patient's family. Follow-up was $100 \%$ complete.

\section{Statistical analyses}

Data were presented as mean \pm s.d. when normally distributed, median (25th-75th percentile) when not-normally distributed, or number (frequency). Comparisons across groups were made using unpaired two-sided $t$-test, the $\chi^{2}$ test, Mann-Whitney $U$ test, Kruskal-Wallis test or analysis of variance, where appropriate. Correlations between serum level of TT or eFT and New York Heart Association (NYHA) class, LVEF or NT-pro-BNP were assessed by Spearman's correlation coefficient. The association of total and free testosterone levels with all cause mortality was determined by Cox proportional hazards regression.

In order to estimate the effect of the serum levels of TT and eFT on 5-year survival rates, Kaplan-Meier curves for cumulative survival were constructed for patients in low, medium and high tertiles according to TT and eFT levels, respectively. Differences in survival rates were tested with the Cox-Mantel log-rank test.

All tests were performed using the statistical software package SPSS (version 15.0.1; SPSS Inc., Chicago, IL, USA).

\section{RESULTS}

\section{Baseline clinical characteristics}

Table 1 shows the baseline clinical characteristics of the entire cohort and of the cohort divided into tertiles based on the LVEF. Mean age at baseline was $68.5 \pm 5.4$ years. Patients in lower tertiles of LVEF were characterized by older age, advanced NYHA class, decreased serum TT and eFT levels, increased SHBG concentration, decreased hemoglobin and plasma albumin concentration, decreased estimated glomerular filtration rate and increased serum NT-pro-BNP level. There were no significant differences among the three tertiles with regard to etiology (ischemic $v s$. no ischemic), mean systolic blood pressure, prevalence of comorbidities (atrial fibrillation, hypertension and diabetes) or medication use (angiotensin-converting enzyme inhibitor or angiotensin II receptor-1 blocker, $\beta$-blocker, digoxin and diuretics).

\section{Prevalence of androgens deficiency}

The median level of TT in all 175 patients was $20.3 \mathrm{nmol}^{-1}$ (25th-75th percentile: $\left.17.0-27.2 \mathrm{nmol} \mathrm{l}^{-1}\right)$, and that of eFT was $0.28 \mathrm{nmol} \mathrm{l}^{-1}$ (25th-75th percentile: $0.21-0.35 \mathrm{nmol} \mathrm{dl}^{-1}$ ) (Table 1). Using the age-specific tenth percentiles of TT and eFT in healthy men in our laboratory as aforementioned as cutoff points, the prevalences of TT and eFT deficiency were $21.7 \%(38 / 175)$ and $27.4 \%$ (48/175), respectively. Of the 175 patients, 17 had a TT level below $8 \mathrm{nmol} \mathrm{l}^{-1}(230$ $\left.\mathrm{ng} \mathrm{\textrm {dl } ^ { - 1 }}\right), 27$ had an eFT level below $0.225 \mathrm{nmol}^{-1}\left(65 \mathrm{pg} \mathrm{ml}^{-1}\right)$ and 12 had both a TT level below $8 \mathrm{nmol}^{-1}$ and an eFT level below $0.225 \mathrm{nmol} \mathrm{l}^{-1}$. Using the criteria for initiation of testosterone treatment as proposed by the current recommendations, ${ }^{21,22}$ that is, either a TT level below $8 \mathrm{nmol}^{-1}\left(230 \mathrm{ng} \mathrm{dl}^{-1}\right)$ or an eFT level below $0.225 \mathrm{nmol} \mathrm{l}^{-1}\left(65 \mathrm{pg} \mathrm{ml}^{-1}\right)$, regardless of age, $32 \%(56 / 175)$ of the patients would be suggested to have hypogonadism by laboratory diagnosis.

\section{Serum androgen level and CHF severity and prognosis}

To assess the relationship between serum androgen level and CHF severity, correlation coefficients between serum level of TT or eFT and NYHA class, LVEF and plasma NT-pro-BNP were computed. There was no significant correlation between TT or eFT level and NYHA class ( $r=-0.12, P=0.14$ and $r=-0.09, P=0.24$, respectively), but both TT and eFT levels were significantly correlated with LVEF ( $r=0.30, P<0.01$ and $r=0.31, P<0.01$, respectively) and NT-pro-BNP ( $r=-0.21, P=0.005$ and $r=-0.23, P=0.003$, respectively).

During a median follow-up period of 3.46 years (25th-75th percentile: 3.01-3.90 years) for survivors, 54 patients (30.9\%) died. The crude mortality risks in tertiles (from low to high) of free testosterone were $46.6 \%, 24.1 \%$ and $22.0 \%$. Figure 1 shows the Kaplan-Meier curves for cumulative survival rate for patients in low, medium and high tertiles according to TT and eFT levels $\left(\chi^{2}=11.82, P=0.003\right.$ and $\chi^{2}=9.60, P=0.008$, respectively).

Using the age-specific criteria for TT and eFT deficiency defined in our laboratory, the crude mortalities in patients with and without TT deficiency were $39.5 \%(15 / 38)$ and $28.5 \%$ (39/137), respectively $\left(\chi^{2}=1.69, P=0.19\right)$, and in patients with and without eFT deficiency, they were $47.9 \%(23 / 48)$ and $24.4 \%(31 / 127)$, respectively $\left(\chi^{2}=9.02\right.$, $P=0.003)$. In additional analysis, using the criteria for initiating testosterone treatment for males as proposed by the current recommendations, ${ }^{21,22}$ the crude mortality in patients who had either a TT level below $8 \mathrm{nmoll}^{-1}$ or an eFT level below $0.225 \mathrm{nmoll}^{-1}$ was $42.9 \%$ (24/ $56)$, as compared the mortality of $25.2 \%(30 / 119)$ in those patients who met neither of the criteria $\left(\chi^{2}=5.56, P=0.023\right)$.

\section{Multivariable analyses}

Table 2 shows the results of multivariable Cox regression, with survival time as the dependent variable, and confounding clinical and laboratory parameters listed in Table $\mathbf{1}$ as independent variables. After adjustment for other variables, only age, LVEF and NYHA class 
Table 1 Baseline characteristics of study patients

\begin{tabular}{|c|c|c|c|c|}
\hline & \multirow{2}{*}{$\begin{array}{c}\text { All patients } \\
(\mathrm{n}=175)\end{array}$} & \multicolumn{3}{|c|}{ Tertiles of $L V E F$} \\
\hline & & Low $(n=58)$ & Medium $(n=58)$ & $\operatorname{High}(\mathrm{n}=59)$ \\
\hline \multicolumn{5}{|l|}{ Clinical variables } \\
\hline Age, year & $68.5 \pm 5.4$ & $69.1 \pm 5.3$ & $68.4 \pm 5.8$ & $67.9 \pm 5.2$ \\
\hline \multicolumn{5}{|l|}{ NYHA class, $n(\%)$} \\
\hline $1-2$ & $112(64.0)$ & 13 (11.6) & $40(35.7)$ & $59(52.7)$ \\
\hline $3-4$ & $63(36.0)$ & $35(55.6)$ & $19(30.2)$ & $9(14.3)$ \\
\hline $\operatorname{LVEF}(\%)$ & $35.6(2.4)$ & $31.3(3.1)$ & $35.9(1.9)$ & $39.6(2.4)$ \\
\hline Systolic blood pressure, $\mathrm{mmHg}$ & $120.2 \pm 14.8$ & $119.4 \pm 15.4$ & $118.6 \pm 14.5$ & $122 \pm 14.6$ \\
\hline Ischemic etiology, $n(\%)$ & $96(54.9)$ & $29(50.0)$ & $33(56.9)$ & $34(57.6)$ \\
\hline \multicolumn{5}{|l|}{ Comorbidities, $n(\%)$} \\
\hline Atrial fibrillation & $36(20.6)$ & $14(23.7)$ & $12(20.6)$ & $10(17.2)$ \\
\hline Hypertension & $97(55.4)$ & $31(53.4)$ & $30(51.7)$ & $36(61.0)$ \\
\hline Diabetes & $32(18.3)$ & $13(22.4)$ & $9(15.5)$ & $10(16.9)$ \\
\hline \multicolumn{5}{|l|}{ Medication, $n(\%)$} \\
\hline ACE inhibitor or ARB & $158(90.3)$ & $53(91.4)$ & $55(94.8)$ & $50(84.7)$ \\
\hline$\beta$-blocker & $119(68.0)$ & $40(69.0)$ & $38(65.5)$ & $41(69.5)$ \\
\hline Digoxin & $62(35.4)$ & $21(36.2)$ & $20(34.5)$ & $21(35.6)$ \\
\hline Diuretic & $159(90.9)$ & $54(93.1)$ & $51(87.9)$ & $54(91.5)$ \\
\hline \multicolumn{5}{|l|}{ Laboratory variables } \\
\hline Estimated GFR, (ml min ${ }^{-1}$ per $\left.1.73 \mathrm{~m}^{2}\right)$ & $66.0 \pm 22.3$ & $62.6 \pm 23.5$ & $66.3 \pm 22.3$ & $69.2 \pm 21.2$ \\
\hline NT-pro-BNP, pg ml ${ }^{-1}$ & $1854(386-3474)$ & $2442(648-4468)$ & $1816(474-3642)$ & $1168(288-2258)$ \\
\hline Plasma albumin $\left(\mathrm{g} \mathrm{I}^{-1}\right)$ & $41.4 \pm 4.6$ & $40.5 \pm 5.5$ & $41.6 \pm 3.8$ & $41.8 \pm 4.1$ \\
\hline Hemoglobin $\left(\mathrm{g}^{-1}\right)$ & $13.4 \pm 1.9$ & $13.2 \pm 2.0$ & $13.5 \pm 1.9$ & $13.6 \pm 1.9$ \\
\hline $\mathrm{TT}\left(\mathrm{nmol} \mathrm{I}^{-1}\right.$ ), median (25th-75th percentile) & $20.3(17.0-27.2)$ & $17.8(11.6-22.8)$ & $20.4(14.2-26.8)$ & $24.0(18.4-32.6)$ \\
\hline eFT $\left(\mathrm{nmol} \mathrm{I}^{-1}\right.$ ), median (25th-75th percentile) & $0.28(0.21-0.35)$ & $0.25(0.12-0.21)$ & $0.28(0.18-0.34)$ & $0.31(0.22-0.44)$ \\
\hline SHBG (nmol I ${ }^{-1}$ ), median (25th-75th percentile) & $56.4(36.8-68.2)$ & $61.2(42.4-76.4)$ & $54.8(38.8-62.2)$ & $48.8(34.6-56.0)$ \\
\hline
\end{tabular}

Abbreviations: ACE, angiotensin-converting enzyme; ARB, angiotensin II receptor-1 blocker; eFT, estimated free testosterone; GFR, glomerular filtration rate; LVEF, left ventricular ejection fraction; NT-pro-BNP, N-terminal pro-brain natriuretic peptide; NYHA, New York Heart Association; SHBG, sex hormone-binding globulin; TT, total testosterone.

were significantly associated with survival time. Although in univariable analyses or after adjustment for age, both serum TT and eFT levels were associated with patient survival time, after adjustment for the full variables, their prognostic significances were abolished (for TT, $\mathrm{OR}=0.97,95 \% \mathrm{CI}: 0.84-1.12, P=0.279$; for $\mathrm{eFT}, \mathrm{OR}=0.92,95 \% \mathrm{CI}$ : $0.82-1.06, P=0.135)$.

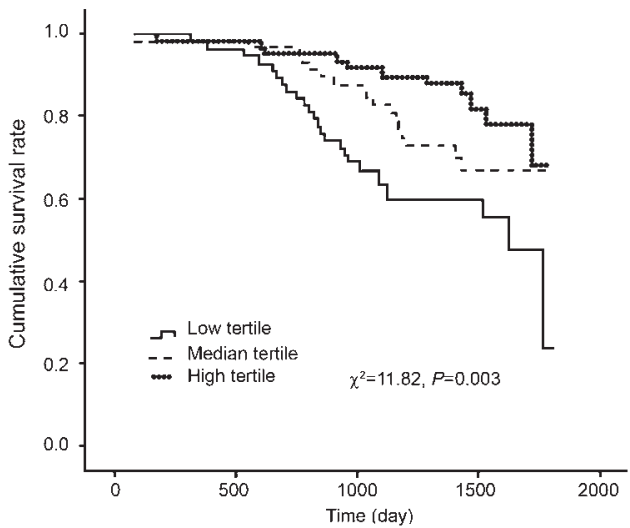

\section{DISCUSSION}

There were two major findings in our present study. First, in an unselected cohort of older men with systolic CHF, we found a relatively high prevalence of reduced serum concentrations of androgens. Serum TT and eFT levels were below the tenth percentile of their healthy peers in $21.7 \%$ and $27.4 \%$ of patients, respectively. Using the criteria for b

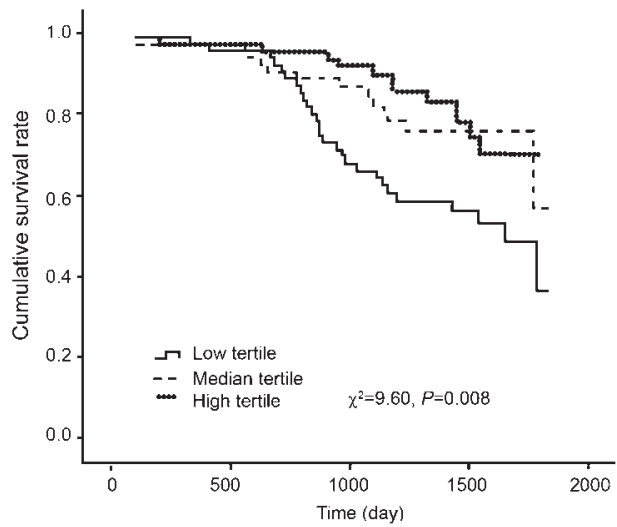

Figure 1 Kaplan-Meier curves reflecting 5-year cumulative survival rates in patients with systolic chronic heart failure according to tertiles of serum total testosterone (a) and serum free testosterone (b). (a) Tertiles of serum total testosterone were divided into low $\left(<15.6 \mathrm{nmol} \mathrm{dl}^{-1}\right)$, medium $\left(15.7-25.8 \mathrm{nmol} \mathrm{dl}^{-1}\right)$ and high $\left(\geqslant 25.9 \mathrm{nmol} \mathrm{dl}^{-1}\right)$. There was significant difference in survival rate among tertiles of serum total testosterone $\left(\chi^{2}=11.82, P=0.003\right.$, by log-rank test). (b) Similarly,

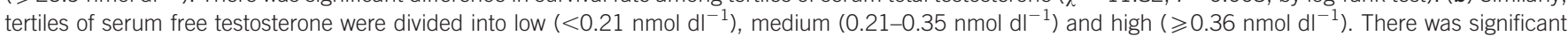
difference in survival rate among tertiles of serum free testosterone $\left(\chi^{2}=9.60, P=0.008\right.$, by log-rank test). 
Table 2 Adjusted hazard ratios for all-cause mortality

\begin{tabular}{llll}
\hline & $H R^{a}$ & $95 \%$ Cl for HR & P value \\
\hline Age, per year & 1.07 & $1.01-1.13$ & 0.034 \\
NYHA class, per 1 advanced class & 1.97 & $1.37-2.82$ & 0.000 \\
LVEF, per $1 \%$ & 0.94 & $0.90-0.98$ & 0.002 \\
TT, per $1 \mathrm{nmol} \mathrm{I}{ }^{-1}$ & 0.97 & $0.84-1.12$ & 0.279 \\
eFT, per $0.01 \mathrm{nmol} \mathrm{I}^{-1}$ & 0.92 & $0.82-1.06$ & 0.135 \\
SHBG, per $1 \mathrm{nmol} \mathrm{I}$ & 1.08 & $0.88-1.26$ & 0.246 \\
\hline
\end{tabular}

Abbreviations: $\mathrm{Cl}$, confidence interval; eFT, estimated free testosterone; $\mathrm{HR}$, hazard ratio; LVEF, left ventricular ejection fraction; NYHA, New York Heart Association; SHBG, sex hormone-binding globulin; TT, total testosterone.

${ }^{\text {a }}$ HRs were adjusted for all variables listed in Table 1.

initiating testosterone treatment for males as proposed by the current recommendations, ${ }^{21,22}$ nearly one-third of our patients would be considered to have laboratory diagnosed late-onset hypogonadism. In addition, both serum TT and eFT levels were correlated with LVEF and plasma NT-pro-BNP level, suggesting the relationship between androgen level and CHF severity; second, we had shown that in men with stable CHF and reduced LVEF, lower levels of serum TT and eFT were associated with poor prognosis. During the mean follow-up time of about 3 and half years, patients with testosterone deficiency, whether defined by our local laboratory criteria, or by current guideline criteria, had higher mortality than those patients without testosterone deficiency. However, these associations were not independent to other conventional clinical prognostic indicators.

Androgen deficiency is known to occur in several chronic disorders as well as in aging, ${ }^{23,24}$ and may even play a role in the pathogenesis of cardiovascular disease. ${ }^{25}$ Some studies have reported low plasma levels of testosterone in patients with $\mathrm{CHF}$, whereas others have not. ${ }^{6} \mathrm{~A}$ major problem in making comparisons between studies is the definition of testosterone deficiency. In recently published recommendations for diagnosis and treatment of late-onset hypogonadism, a TT less than $230 \mathrm{ng} \mathrm{dl}^{-1}$ was suggested as a lower normal range and a free testosterone level below $225 \mathrm{pmol}^{-1}\left(6.5 \mathrm{ng} \mathrm{dl}^{-1}\right)$ was suggested as thresholds when considering testosterone supplementation. ${ }^{21,22}$ In the present study, we defined testosterone deficiency as below the tenth percentile for TT and eFT levels in age groups from a large population of 1080 healthy male subjects. ${ }^{18}$ Because age is one of the major determinants of testosterone level in men, ${ }^{18,23,24}$ we think that a definition of testosterone deficiency should not be based on threshold values that are constant across the entire age spectrum.

We found associations of decrease in serum TT and eFT levels with reduced LVEF or increased NT-pro-BNP, but not with NYHA class. These findings are in contrast with results reported by Jankowska et al., ${ }^{15}$ who found that serum TT and eFT levels correlated inversely with NYHA class in men with CHF, but neither TT nor eFT level correlated with echocardiographic or humoral indices of left ventricle function. As further comparisons, in patients with systolic and diastolic heart failure, Güder and colleagues ${ }^{16}$ found that free testosterone, but not TT, was inversely associated with NYHA class and plasma NT-pro-BNP. The divergent and equivocal results, as well as the rather weak, although statistically significant correlations, suggest that serum androgen levels are not good surrogate of disease severity in $\mathrm{CHF}$ patients.

Until now, two studies have investigated the prognostic significance of androgens in heart failure. In a study of 208 men with CHF, Jankowska et al. ${ }^{15}$ reported that TT and eFT, as well as DHEAS and IGF-1 levels, were predictive of mortality, independent of age, LVEF,
NYHA class, GRF and pro-BNP levels. More recently, Güder et al., ${ }^{16}$ in their multivariable analyses of 191 patients with $\mathrm{CHF}$, found that after adjustment of variables that were associated with both the exposure and the outcome, neither TT nor eFT, DHEAS or SHBG represent independent predictors of mortality risk. The results of our present study are similar to the findings of Güder et al. ${ }^{16}$ Although both serum TT and eFT levels were associated with patient survival time in univariable analyses, after adjustment for confounding factors, their prognostic significances were abolished. Our results, as well as those reported by Güder et al., ${ }^{16}$ suggest that androgens are unlikely to play a causal role in the progression of heart failure.

In spite of the uncertain role of testosterone in the pathophysiology of CHF, several clinical trials have examined the effect of testosterone therapy in $\mathrm{CHF}$ and have showed promising results. In an uncontrolled open-label trial, Tomoda found that treatment with oxymetholone improved cardiac function. ${ }^{10}$ In a small double-blinded study, Pugh et al. ${ }^{11}$ showed that intramuscular testosterone injections improved quality of life and functional capacity in CHF patients. As a follow-up to this pilot study, Malkin et al. ${ }^{12}$ demonstrated similar effects of testosterone patches. More recently, in a double-blind, placebo-controlled, randomized trial in 70 elderly patients with CHF, Caminiti et al. ${ }^{13}$ showed that intramuscular testosterone supplementation on top of optimal therapy improves functional exercise capacity, muscle strength, insulin and baroreflex sensitivity. These therapeutic effects were also observed in female patients. ${ }^{14}$ Therefore, testosterone treatment may represent a new therapeutic approach in CHF that might have some potential.

Several limitations of the present study should be mentioned. First, the results are based on a single testosterone measurement in the study subjects, which may inadequately reflect the average sex hormone release. However, the distributions of both TT and eFT levels found in this study were consistent with our previous reports of healthy men; second, the modest sample size may have limited the power of the analyses; third, we used electrochemiluminescence for measuring serum testosterone levels, rather than the current mass spectrometry-based method as recommended by the Endocrine Society; ${ }^{26}$ therefore, TT concentration might be overestimated in our study, as reflected in the relatively higher levels of TT and eFT compared to other published reports; fourth, our study population did not include all the consecutive patients who met the study criteria, and the information regarding patients who refused to participate had not been collected; this may possibly lead to bias in our analysis.

In summary, our present study showed that in men with systolic heart failure, androgen deficiency was relatively common and low TT or eFT levels were associated with disease severity and long-term mortality. However, the prognostic utility of TT and eFT depended on other clinical factors, suggesting that androgens are unlikely to play a causal role in the progression of heart failure, and the mechanisms of the reported beneficial effects of testosterone treatment for $\mathrm{CHF}$ patient remain to be elucidated.

\section{AUTHOR CONTRIBUTIONS}

HYW designed the study, performed the statistical analysis and drafted the manuscript. XFW and JHW participated in patient enrollment, collection of clinical data and helped to draft the manuscript. JYL participated in the study design and measurement of serum concentrations of androgens. All authors read and approved the final manuscript.

\section{COMPETING FINANCIAL INTERESTS}

The authors declared no competing financial interests. 


\section{ACKNOWLEDGMENTS}

This research was supported in part by a special grant from the Minister of Health of China (No. 06H050).

1 Ammar KA, Jacobsen SJ, Mahoney DW, Kors JA, Redfield MM et al. Prevalence and prognostic significance of heart failure stages: application of the American College of Cardiology/American Heart Association heart failure staging criteria in the community. Circulation 2007; 115: 1563-70.

2 Gu DF, Huang GY, He J. Investigation of prevalence and distributing feature of chronic heart failure in Chinese adult population. Chin J Cardio/ 2003; 31: 3-6.

3 Anker SD, Chua TP, Ponikowski P, Harrington D, Swan JW et al. Hormonal changes and catabolic/anabolic imbalance in chronic heart failure and their importance for cardiac cachexia. Circulation 1997; 96: 526-34

4 Moriyama Y, Yasue H, Yoshimura M, Mizuno Y, Nishiyama K et al. The plasma levels of dehydroepiandrosterone sulfate are decreased in patients with chronic heart failure in proportion to the severity. J Clin Endocrinol Metab 2000; 85: 1834-40.

5 Kontoleon PE, Anastasiou-Nana MI, Papapetrou PD, Alexopoulos G, Ktenas V et al. Hormonal profile in patients with congestive heart failure. Int J Cardiol 2003; 87 179-83.

6 Sacca L. Heart failure as a multiple hormone deficiency syndrome. Circ Heart Fail 2009; 2: 151-6.

7 Anker SD, Clark AL, Kemp M, Salsbury C, Teixeira MM et al. Tumor necrosis factor and steroid metabolism in chronic heart failure: possible relation to muscle wasting. J Am Coll Cardiol 1997; 30: 997-1001.

8 Iellamo F, Rosano G, Volterrani M. Testosterone deficiency and exercise intolerance in heart failure: treatment implications. Curr Heart Fail Rep 2010; 7: 59-65.

9 Malkin CJ, Channer KS, Jones TH. Testosterone and heart failure. Curr Opin Endocrinol Diabetes Obes 2010; 17: 262-8.

10 Tomoda $\mathrm{H}$. Effect of oxymetholone on left ventricular dimensions in heart failure secondary to idiopathic dilated cardiomyopathy or to mitral or aortic regurgitation. Am J Cardio/1999; 83: 123-5.

11 Pugh PJ, Jones RD, West JN, Jones TH, Channer KS. Testosterone treatment for men with chronic heart failure. Heart 2004; 90: 446-7.

12 Malkin CJ, Pugh PJ, West JN, van Beek EJ, Jones TH et al. Testosterone therapy in men with moderate severity heart failure: a double-blind randomized placebo controlled trial. Eur Heart J 2006; 27: 57-64.

13 Caminiti G, Volterrani M, lellamo F, Marazzi G, Massaro R et al. Effect of long-acting testosterone treatment on functional exercise capacity, skeletal muscle performance, insulin resistance, and baroreflex sensitivity in elderly patients with chronic heart failure a double-blind, placebo-controlled, randomized study. J Am Coll Cardiol 2009; 54: 919-27.

14 lellamo F, Volterrani M, Caminiti G, Karam R, Massaro R et al. Testosterone therapy in women with chronic heart failure: a pilot double-blind, randomized, placebocontrolled study. J Am Coll Cardiol 2010; 56: 1310-6.

15 Jankowska EA, Biel B, Majda J, Szklarska A, Lopuszanska M et al. Anabolic deficiency in men with chronic heart failure: prevalence and detrimental impact on survival. Circulation 2006; 114: 1829-37.

16 Güder G, Frantz S, Bauersachs J, Ertl G, Angermann CE et al. Low circulating androgens and mortality risk in heart failure. Heart 2010; 96: 504-9.

17 Laghi F, Antonescu-Turcu A, Collins E, Segal J, Tobin DE et al. Hypogonadism in men with chronic obstructive pulmonary disease: prevalence and quality of life. Am J Respir Crit Care Med 2005; 171: 728-33.

18 Li JY, Li XY, Li M, Zhang GK, Ma FL et al. Decline of serum levels of free testosterone in aging healthy Chinese men. Aging Male 2005; 8: 203-6.

19 Vermeulen A, Verdonck L, Kaufman JM. A critical evaluation of simple methods for the estimation of free testosterone in serum. J Clin Endocrinol Metab 1999; 84: 3666 72.

20 Levey AS, Bosch JP, Lewis JB, Greene T, Rogers N et al. A more accurate method to estimate glomerular filtration rate from serum creatinine: a new prediction equation. Modification of Diet in Renal Disease Study Group. Ann Intern Med 1999; 130: 46170.

21 Wang C, Nieschlag E, Swerdloff R, Behre HM, Hellstrom WJ et al. Investigation, treatment, and monitoring of late-onset hypogonadism in males: ISA, ISSAM, EAU, EAA, and ASA recommendations. J Androl 2009; 30: 1-9.

22 Bhasin S, Cunningham GR, Hayes FJ, Matsumoto AM, Snyder PJ et al. Testosterone therapy in adult men with androgen deficiency syndromes: an endocrine society clinical practice guideline. J Clin Endocrinol Metab 2006; 91: 1995-2010.

23 Araujo AB, Kupelian V, Page ST, Handelsman DJ, Bremner WJ et al. Sex steroids and all-cause and cause-specific mortality in men. Arch Intern Med 2007; 167: 1252 60.

24 Matsumoto AM. Andropause: clinical implications of the decline in serum testosterone levels with aging in men. J Gerontol A Biol Sci Med Sci 2002; 57 M76-99.

25 Corona G, Mannucci E, Forti G, Maggi M. Hypogonadism, ED, metabolic syndrome and obesity: a pathological link supporting cardiovascular diseases. Int J Androl 2009; 32 587-98.

26 Rosner W, Auchus RJ, Azziz R, Sluss PM, Raff H. Utility, limitations, and pitfalls in measuring testosterone: an Endocrine Society position statement. J Clin Endocrinol Metab 2007; 92: 405-13. 